\title{
Glycosaminoglycan chains of biglycan promote bone morphogenetic protein-4-induced osteoblast differentiation
}

\author{
YAPING YE, WEIHUA HU, FENGJING GUO, WEIKAI ZHANG, JIANG WANG and ANMIN CHEN \\ Department of Orthopedics, Tongji Hospital, Tongji Medical College, \\ Huazhong University of Science and Technology, Wuhan, Hubei, P.R. China
}

Received May 16, 2012; Accepted July 2, 2012

DOI: $10.3892 /$ ijmm.2012.1091

\begin{abstract}
Biglycan (BGN) has been reported to promote bone morphogenetic protein-4 (BMP-4) stimulated osteoblastic differentiation. However, the underlying mechanism has yet to be fully elucidated. The glycosaminoglycan (GAG) chains of BGN have a variety of biological functions. In the present study, we explored the potential role of the GAG chains of $\mathrm{BGN}$ in promoting BMP-4-induced osteoblast differentiation. BGN knockout (KO) murine calvarial cells were transfected with adenovirus overexpressing wild-type BGN (Adv-BGN), adenovirus expressing GAG-mutant BGN (Adv-BGNm) and adenovirus without BGN (Adv-Emp). Transfected cells were treated with or without BMP-4. Subsequently, BMP-4 signaling and function were assessed by evaluating the expression of the osteoblast differentiation-related proteins, Smad1/5/8 phosphorylation and alkaline phosphatase (ALP) activity. Furthermore, the binding specificity of the transfected cells to BMP-4 was also investigated using immunofluorescence staining. Our study demonstrated that a mutant BGN lacking GAG chains decreased BGN-assisted BMP-4 signaling and osteoblast differentiation and that the expression of this mutant BGN in biglycan knockout (BGN-KO) calvarial osteoblasts could not rescue its differentiation deficiency as efficiently as wild-type (WT) BGN. These results strongly suggest that the GAG chains of BGN promote BGN-assisted BMP-4 function.
\end{abstract}

\section{Introduction}

The extracellular matrix (ECM) of bone, which functions largely to provide biomechanical strength, is a complex network composed of heterogeneous macromolecules, including a number of small leucine-rich proteoglycans

Correspondence to: Dr Weihua Hu, Department of Orthopedics, Tongji Hospital, Tongji Medical College, Huazhong University of Science and Technology, 1095 JieFang Avenue, Wuhan, Hubei 430030, P.R. China

E-mail:whhu@tjh.tjmu.edu.cn

Key words: glycosaminoglycan chains, biglycan, bone morphogenetic protein-4, osteoblast differentiation
(SLRPs) (1). Biglycan (BGN), a member of the SLRP family, consists of a $45 \mathrm{kDa}$ protein core and 2 glycosaminoglycan (GAG) chains, chondroitin sulfate (CS) and dermatan sulfate (DS), which are covalently linked to the protein core (2). The CS/DS chains are attached at amino acids 5 and 10 in the human BGN core protein (3). BGN is highly expressed in the $\mathrm{ECM}$ of bone and is localized on the surface of osteoblasts (4).

BGN is multifunctional and is widely involved in many biological processes. A noteble discovery is that BGN promotes osteoblast differentiation. BGN knockout (KO) mice have an age-dependent osteoporosis-like phenotype including a reduced growth rate, lower bone mass due to decreased bone formation and significantly shortened femurs (5-7). BGN modulates osteoblast differentiation by regulating bone morphogenetic protein-4 (BMP-4) signaling. Chen et al (8) were the first to propose that BGN modulates BMP-4-induced signaling to control osteoblast differentiation. Their results showed that BGN deficiency affected BMP-4 signal transduction, thus reducing core-binding factor $\alpha 1$ (Cbfa1) expression and in turn causing defective osteoblast differentiation. BGN plays an important role as a reservoir for balancing the growth factor activity of BMP-4 to control osteoblast differentiation. The absence of BGN caused less BMP-4 binding and subsequently reduced the sensitivity of osteoblasts to BMP-4 stimulation, which ultimately led to a defect in the differentiation of osteoblasts (8). This function could be attributed to the ability of BGN to bind BMP-4 and other molecules $(8,9)$. However, the domains of BGN that are involved in its positive modulation of BMP-4 function remain unknown.

Recent research has focused on the GAG chains of BGN. GAGs are highly negatively charged polysaccharides that maintain the structural integrity and viscosity of the extracellular environment $(10,11)$. GAGs have been reported to be associated with a variety of amyloid deposits $(12,13)$. The DS GAGs of decorin are involved in the organization of the collagen fibrils (14). Furthermore, GAGs affect cell growth in both malignant and normal cells of the osteoblastic lineage in a concentration-dependent manner (15). GAGs contribute to numerous regulatory molecular networks through binding a variety of signaling molecules, including cytokines, chemokines, growth factors and differentiation factors (16). The present study investigated the role of the GAGs of BGN in BGN-assisted BMP-4 function using BGN-KO calvarial osteoblastic cells. 


\section{Materials and methods}

Animals. Wild-type (WT) and biglycan knockout (BGN-KO) male mice (C57B6/129) were purchased from the SLAC Laboratory Animal Center (Shanghai, China). All animal care and experimental procedures were approved by the Ethics Committee of Tongji Medical College, Huazhong University of Science and Technology. Mice were housed in polypropylene cages $(32 \times 40 \times 18 \mathrm{~cm})$ under controlled temperature $\left(22-24^{\circ} \mathrm{C}\right)$ and humidity with a 12 -h light/dark cycle and free access to food and water. All experiments were performed using WT and BGN-KO mice (1-5 days old). The genotypes of the WT and BGN-KO mice were confirmed by polymerase chain reaction (PCR) as previously described (7).

Reagents. Human recombinant BMP-4 (hrBMP-4) was purchased from R\&D Systems, Inc. (Minneapolis, MN, USA). The bicinchoninic acid (BCA) protein detection kit, chemiluminescent substrate kit and FITC-conjugated goat anti-rabbit secondary antibody were purchased from Pierce Chemical Co. (Thermo Fisher Scientific, Waltham, MA, USA). The alkaline phosphatase (ALP) kit was purchased from Sigma Chemical Co. (Sigma-Aldrich, USA). CLS-2 bacterial collagenase was purchased from Worthington (Lakewood, USA).

Culture medium. The complete medium consisted of $\alpha$-modified minimum essential medium (Gibco-BRL, USA) supplemented with glutamine $(2 \mathrm{mM})$, penicillin $(100 \mathrm{U} / \mathrm{ml})$, streptomycin $(100 \mu \mathrm{g} / \mathrm{ml})$ (Sigma), 2-mercaptoethanol $(0.1 \mathrm{mM})$ and $10 \%$ fetal bovine serum (Gibco-BRL). The differentiation medium consisted of the complete medium supplemented with $2 \mathrm{mM} \beta$-glycerophosphate and $0.1 \mathrm{mM}$ L-ascorbic acid phosphate magnesium (Sigma).

Preparation of murine calvarial cells. Neonatal murine calvarial cells were prepared as previously described (17). Briefly, calvariae harvested from WT or BGN-KO mice (1-5 days old) were pretreated with $4 \mathrm{mM}$ EDTA in phosphate-buffered saline (PBS) for 2-10 $\mathrm{min}$. The calvariae were digested with CLS-2 bacterial collagenase at $200 \mathrm{U} / \mathrm{ml}$ in PBS for 5-10 min. Cells from the last 3 digestions were collected and served as the starting population of highly enriched osteoblastic cells (18). The cells were washed twice with complete culture medium, seeded into 6-well plates at a density of $1,000-2,000$ cells $/ \mathrm{cm}^{2}$ and cultured at $37^{\circ} \mathrm{C}$ in a humidified atmosphere containing $5 \% \mathrm{CO}_{2}$ in the presence or absence of BMP-4 (30 ng/ml). Cultures were fed with the differentiation medium twice a week once they reached confluence ( $\sim 7$ days). The cells were then collected from cultures at intervals indicated in the following experiments.

Transfection of $B G N-K O$ cells. Adenovirus strains without the BGN gene (Adv-Emp), overexpressing wildtype BGN (Adv-BGN), or expressing GAG-mutant BGN (Adv-BGNS5AS10A or Adv-BGNm) were purchased from GeneChem (Shanghai, China). The BGN-KO cells were plated on 12-well plates in triplicate at a density of $2 \times 10^{4}$ cells/well in complete medium, cultured to $30 \%$ confluence and transfected either with $3.75 \times 10^{7} \mathrm{PFU} / \mathrm{ml}$ of Adv-BGN, 3.75 $\times 10^{7} \mathrm{PFU} / \mathrm{ml}$ of Adv-BGNm or $3.75 \times 10^{7} \mathrm{PFU} / \mathrm{ml}$ of recombinant adenovirus without the BGN gene/cDNA (Adv-Emp) for $72 \mathrm{~h}$. Then, the transfected cells were tested as specified in the text herein.

Western blot analysis. For western blotting, confluent cells were cultured in medium with $2 \%$ serum overnight and then treated with BMP-4 $(30 \mathrm{ng} / \mathrm{ml})$ for the duration specified. The cells were washed with PBS and lysed in an extraction buffer (25 mM Tris-Cl, pH 7.2, 1\% Triton X-100, 0.1\% SDS, $1 \%$ sodium deoxycholate, $0.1 \mathrm{M} \mathrm{NaCl}$ and $1 \mathrm{mM}$ EDTA) containing a protease inhibitor cocktail. Then, the lysate was mixed with sample buffer containing $50 \mathrm{mM}$ Tris/ $\mathrm{HCl}$ (pH 7.6), 2\% SDS, 10\% glycerol, $10 \mathrm{mM}$ dithiothreitol and $0.2 \%$ bromophenol blue and boiled for $5 \mathrm{~min}$. The protein concentration in the supernatant was determined using the BCA kit according to the manufacturer's instructions. The proteins were separated by SDS-PAGE (10\% gel), transferred to a nitrocellulose membrane and incubated with primary antibody at $4^{\circ} \mathrm{C}$ overnight. Immune complexes were detected with the appropriate secondary antibodies and enhanced chemiluminescence (ECL) and quantitatively analyzed using Kodak Digital Science 1D software (Eastman Kodak Company). The relative intensity was expressed as the total optical density.

Measurement of alkaline phosphatase activity. Confluent cells were incubated for $48 \mathrm{~h}$ in the presence or absence (control) of $30 \mathrm{ng} / \mathrm{ml} \mathrm{BMP-4}$ in serum-free medium and lysed in lysis buffer $(20 \mathrm{mM}$ Tris, $0.5 \mathrm{mM} \mathrm{MgCl}, 0.1 \mathrm{mM} \mathrm{ZnCl}$ and $0.1 \%$ Triton $\mathrm{X}-100$ ). The alkaline phosphatase (ALP) levels in the lysates were determined using an ALP kit and the production of p-nitrophenol was measured by spectrophotometric absorbance at $405 \mathrm{~nm}$. The ALP value was calculated using standards and expressed as Sigma U/mg of protein lysate. One Sigma unit is equal to $1 \mu \mathrm{M}$ p-nitrophenol/h.

Immunofluorescence. Well-cut slides were placed into 12-well plates. Cells were then seeded into each well at $1 \times 10^{4}$ cells/well in complete medium and cultured until confluence. To detect BMP-4 binding, the cells were preincubated with BMP-4 $(10 \mu \mathrm{g} / \mathrm{ml})$ for $2 \mathrm{~h}$ at room temperature and washed with PBS 3 times. The slides were removed from the plates, and the cells were fixed in $4 \%$ phosphate-buffered formaldehyde for $30 \mathrm{~min}$, then washed and blocked in PBS with $0.1 \%$ BSA and $5 \%$ normal goat serum (blocking solution) for $30 \mathrm{~min}$ at room temperature. To identify BMP-4-positive cells, the slides were incubated with anti-BMP-4 antibody at $4^{\circ} \mathrm{C}$ overnight, then washed and incubated with FITC-conjugated goat anti-rabbit secondary antibody for $2 \mathrm{~h}$ at room temperature. The cells were subsequently washed and incubated with Hoechst to stain the nuclei. Slides were then imaged using a fluorescence microscope (Olympus BX51; Tokyo, Japan), and the results were analyzed quantitatively with Image-Pro ${ }^{\circledR}$ Plus (IPP) software.

Statistical analysis. Results are expressed as the mean \pm standard deviation (SD), calculated from 3 independent experiments. The results were analyzed with SPSS 13.0 statistical software. A one-way ANOVA procedure followed by least significant difference post hoc tests was used to determine the statistical significance of differences of the means. 
$\mathrm{P}<0.05$ was considered to indicate statistically significant differences.

\section{Results}

Characterization of adenovirus-mediated expression of wild-type and mutant BGN in BGN-KO osteoblastic cells. To test the adenovirus transfection efficiency and the BGN expression in the osteoblasts, we measured the quantity of BGN produced by osteoblasts in complete medium following adenoviral transfection. To confirm that the BGN expression persisted for at least 2 weeks, BGN synthesis was analyzed at Days 3, 6 and 14 using western blot analysis with specific antibodies (Fig. 1A).

The expression level of BGN in BGN-KO osteoblastic cells transfected with Adv-BGN was highest at Day 6 and began to decrease at Day 14. However, the synthesis of mutant BGN in BGN-KO osteoblastic cells transfected with Adv-BGNm increased continually from Day 3 to 14 . Furthermore, as judged by electrophoretic mobility on SDS-PAGE, BGN produced from Adv-BGN-transfected BGN-KO osteoblastic cells and endogenous BGN produced from WT osteoblasts were processed similarly, suggesting that WT BGN was correctly expressed in Adv-BGN-transfected BGN-KO osteoblastic cells.

Chondroitinase-ABC (C-ABC) lysates $(0.3 \mathrm{U} / 100 \mu \mathrm{g})$ were used to generate deglycated BGN. Following digestion with C-ABC lyases, the BGN produced by Adv-BGN- and Adv-BGNm-transfected BGN-KO cells or WT osteoblasts migrated similar distances on SDS-PAGE (Fig. 1B), which suggested that the mutant BGN lacking GAG chains was correctly expressed in BGN-KO osteoblastic cells.

The expression of mutant BGN in BGN-KO calvarial osteoblastic cells cannot rescue its differentiation deficiency as efficiently as wild-type BGN. Several studies have indicated that Cbfa1 is an osteoblast-specific transcription factor and a regulator of osteoblast differentiation, controlling the expression of specific extracellular matrix proteins and intracellular proteins, including osteopontin, bone sialoprotein (BSP) and osteocalcin (19-21). The expression of Cbfal is downregulated in BGN-KO cells (22). In the present study, we measured Cbfal expression in BGN-KO osteoblastic cells at the protein level by western blot analysis and found that both Adv-BGN and Adv-BGNm transfection enhanced Cbfal protein expression in BGN-KO osteoblastic cells compared with the Adv-Emp control, either with or without BMP-4 treatment. Notably, Cbfal expression in Adv-BGN-transfected osteoblastic cells was significantly upregulated compared to Adv-BGNm-transfected osteoblastic cells after treatment with BMP-4. This result suggested that BGN, particularly its GAG chains, is essential for BMP-4-induced Cbfal expression (Fig. 2A and B).

We also evaluated the expression of osteoblastic proteins in WT and BGN-KO osteoblastic cells transfected with Adv-Emp, Adv-BGN and Adv-BGNm. Osteopontin, BSP and osteocalcin expression were measured by western blot analysis. These results showed that the expression of osteopontin, $\mathrm{BSP}$ and osteocalcin were all downregulated in BGN-KO cells and Adv-Emp-transfected BGN-KO cells compared with

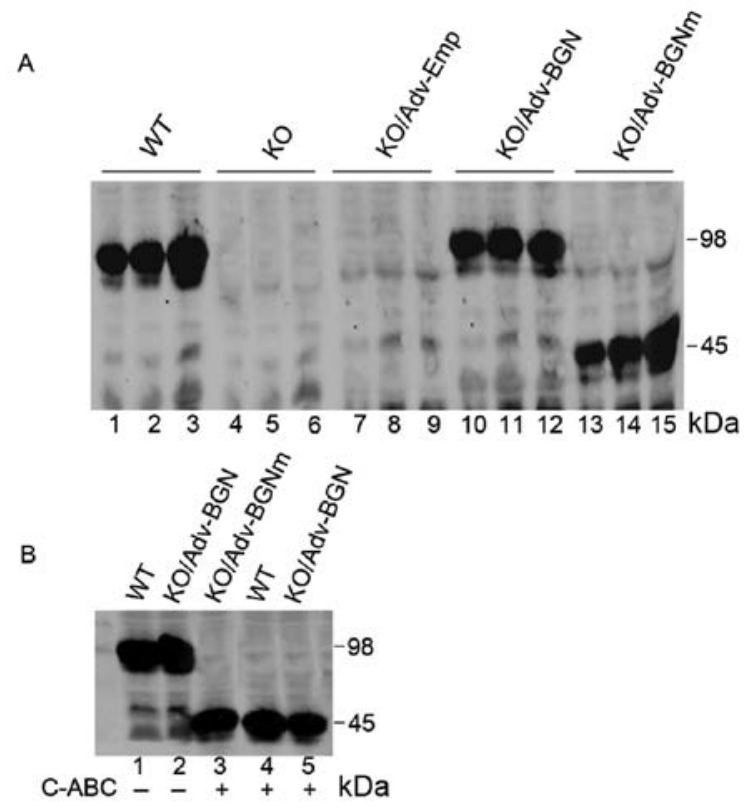

Figure 1. Expression of wild-type BGN and mutant BGN in Adv-BGN and Adv-BGNm-transfected BGN-KO osteoblastic cells was evaluated using SDS-PAGE. (A) Lanes 1-3, positive control: BGN expression in wild-type osteoblasts (Days 3, 6 and 14); lanes 4-6, blank control: BGN expression in BGN-KO osteoblasts (Days 3, 6 and 14); lanes 7-9, negative control: BGN expression in BGN-KO osteoblasts transfected with Adv-Emp (Days 3, 6 and 14); lanes 10-12, BGN expression in BGN-KO osteoblasts transfected with Adv-BGN (Days 3, 6 and 14); and lanes 13-15, BGN expression in BGN-KO osteoblasts transfected with Adv-BGNm (Days 3, 6 and 14). (B) Lane 1, BGN expression in wild-type osteoblasts (Day 6); lane 2, BGN expression in BGN-KO osteoblasts transfected with Adv-BGN (Day 6); lane 3, BGN derived from Adv-BGNm transfected BGN-KO osteoblasts (Day 6) treated with C-ABC lyase; lane 4, BGN derived from wild-type osteoblasts (Day 6) treated with $\mathrm{C}-\mathrm{ABC}$ lyase; and lane 5, BGN derived from Adv-BGNtransfected BGN-KO osteoblasts (Day 6) treated with C-ABC lyase.

WT osteoblastic cells. Both Adv-BGN and Adv-BGNm transfection can rescue the deficiency of these 3 specific proteins in BGN-KO cells. However, the expression of these proteins in the Adv-BGN-transfected group is significantly higher than that in the Adv-BGNm-transfected group (Fig. 2C and D). DM1A was selected as the internal control.

As an early osteogenic differentiation marker, ALP activity was also analyzed using an ALP activity assay (Fig. 3). The ALP level of Adv-BGN-transfected BGN-KO osteoblastic cells treated with BMP-4 increased 1.7-fold (from $33 \pm 1.7$ to $57 \pm 3.0, \mathrm{P}<0.01)$. The ALP level in Adv-BGNmtransfected BGN-KO osteoblastic cells treated with BMP-4 also increased 1.5-fold (from $30 \pm 2.0$ to $45 \pm 2.6, \mathrm{P}<0.01$ ). The ALP level before and after treatment with BMP-4 was highest in WT osteoblastic cells than in all other groups. Collectively, these data suggest that BMP-4-induced ALP expression in BGN-KO osteoblastic cells could be partially rescued by Adv-BGN transfection.

$B G N-K O$ osteoblastic cells transfected with Adv-BGN exhibit significantly greater BMP-4 binding than BGN-KO cells transfected with Adv-BGNm. BGN-KO osteoblastic cells have a low affinity for BMP-4 binding. Therefore, we investigated whether the ectopic expression of BGN or mutant BGN could upregulate the binding of BGN-KO cells to BMP-4. BMP-4positive cells were stained using immunofluorescence. 
A

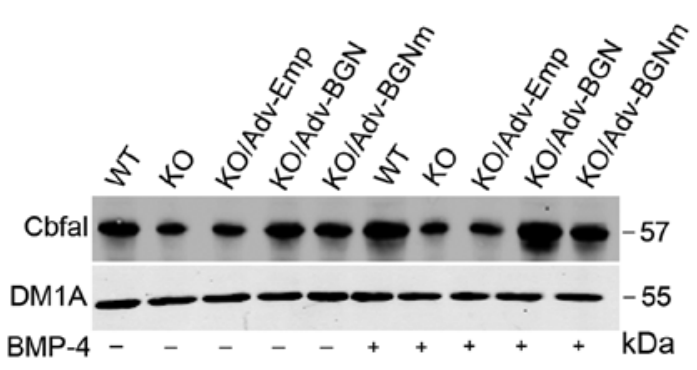

C

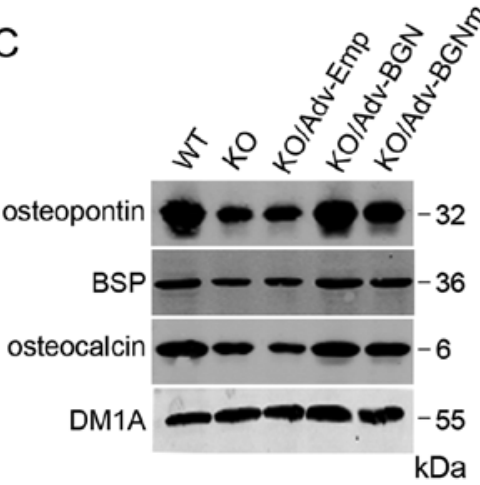

B
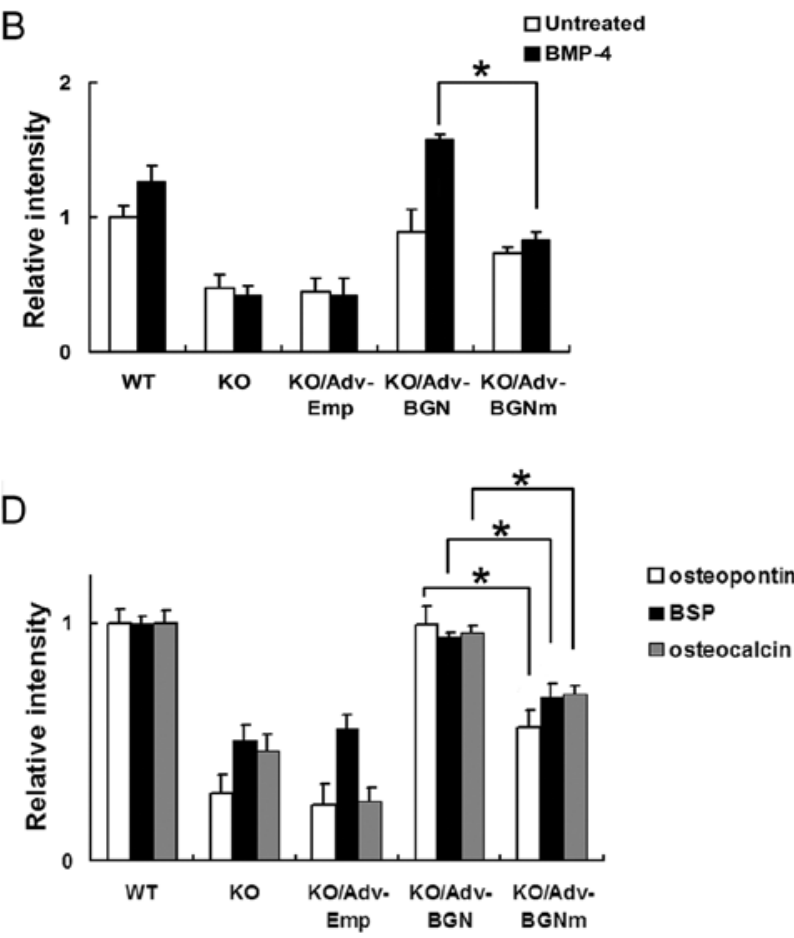

Figure 2. Cbfal and related osteoblastic markers were significantly upregulated in Adv-BGN-transfected osteoblasts. (A and B) Lanes 1-5, Cbfal expression in WT, BGN-KO, Adv-Emp-transfected, Adv-BGN-transfected and Adv-BGNm-transfected osteoblasts without BMP-4 treatment; lanes 6-10, Cbfal expression in WT, BGN-KO, Adv-Emp-transfected, Adv-BGN-transfected and Adv-BGNm-transfected osteoblasts after BMP-4 treatment. Equal protein loading is demonstrated by probing the same blot with a monoclonal antibody against DM1A. The Cbfal expression level in the Adv-BGN-transfected cells is significantly higher than that in the Adv-BGNm-transfected cells ("P<0.05, Adv-BGN/Adv-BGNm). (C and D) The expression of osteopontin, BSP and osteocalcin were detected in WT, BGN-KO, Adv-Emp-transfected, Adv-BGN-transfected and Adv-BGNm-transfected osteoblasts (lanes 1-5) after BMP-4 treatment. Equal protein loading is demonstrated by probing the same blot with a monoclonal antibody against DM1A. The osteopontin, BSP and osteocalcin expression is significantly higher in Adv-BGN-transfected cells than in Adv-BGNm-transfected cells *P<0.05, Adv-BGN/Adv-BGNm.

Specifically, cells were incubated with BMP-4 for $2 \mathrm{~h}$. After washing with PBS, cells were stained with a fluorescently labeled anti-BMP-4 antibody. The BGN-KO osteoblastic cells transfected with Adv-BGN or Adv-BGNm had higher fluorescence intensity than the BGN-KO cells transfected with Adv-Emp (Fig. 4).

However, transfection with Adv-BGNm did not enhance the binding affinity of BGN-KO cells to BMP-4 as effectively as transfection with Adv-BGN. Quantitative analysis showed that the Adv-BGNm-transfected BGN-KO osteoblastic cells had less overall fluorescence intensity ( 25 vs. $40 \%, \mathrm{P}<0.05$ ) than Adv-BGN-transfected cells (Fig. 4). Endogenous BMP-4 was measured prior to the preincubation with BMP-4 and only $<1 \%$ of the untreated BGN-KO cells were BMP-4-positive (data not shown). These results show that the GAG chains of BGN play an important role in the binding of osteoblastic cells to BMP-4 at the cellular level, which may explain the reason why expression of mutant BGN in BGN-KO calvarial osteoblastic cells could not rescue its differentiation deficiency as efficiently as WT BGN.

Effects of $A d v-B G N$ on BMP-4-induced signal transduction in BGN-KO osteoblastic cells. Finally, we examined BMP-4 signal transduction in BGN-KO osteoblastic cells by measuring phosphorylated Smad1/5/8 (P-Smad1/5/8). To determine whether the level of P-Smad1/5/8 in BGN-KO cells could be enhanced by the overexpression of BGN,

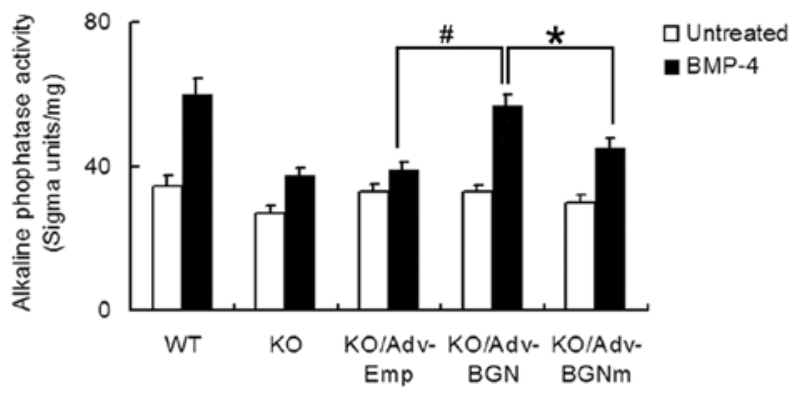

Figure 3. ALP activity was significantly increased in Adv-BGN-transfected BGN-KO osteoblastic cells. ALP activity in Adv-BGN-transfected BGN-KO osteoblastic cells treated with BMP-4 was significantly higher than that in Adv-BGNm- and Adv-Emp-transfected BGN-KO osteoblastic cells treated with BMP-4. ${ }^{*} \mathrm{P}<0.05$, Adv-BGN/Adv-BGNm; ${ }^{*} \mathrm{P}<0.05$, Adv-BGN/Adv-Emp. ALP activity in Adv-Emp-transfected BGN-KO osteoblastic cells and Adv-BGNm-transfected BGN-KO osteoblastic cells treated with BMP-4 was significantly different.

BGN-KO osteoblastic cells were transfected with Adv-Emp, Adv-BGN and Adv-BGNm. Once the cells reached confluence, they were treated with BMP-4 $(30 \mathrm{ng} / \mathrm{ml})$ for $30 \mathrm{~min}$ and $\mathrm{P}-\mathrm{Smad} 1 / 5 / 8$ levels were measured by western blot analysis. We found that the P-Smad1/5/8 level was significantly increased in Adv-BGN-transfected BGN-KO osteoblastic cells treated with BMP-4 compared to Adv-BGNm- or Adv-Emp-transfected BGN-KO osteoblastic cells (Fig. 5). 
A
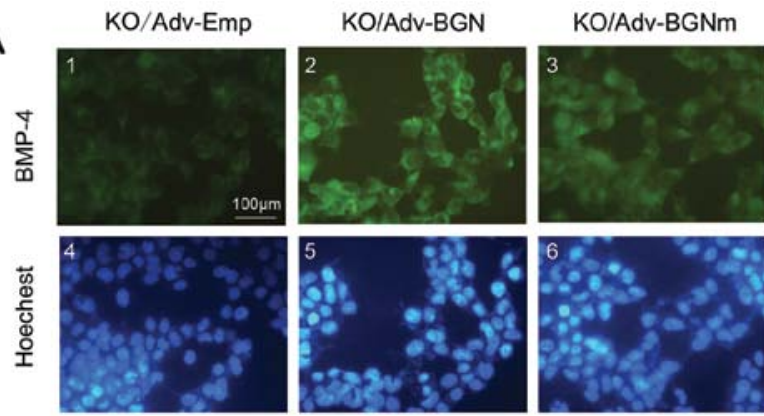

B

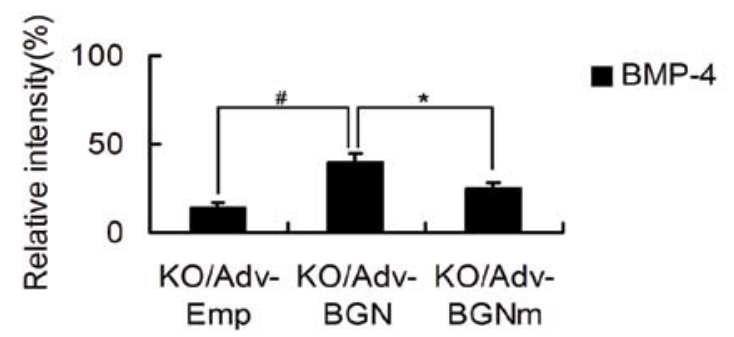

Figure 4. (A) Adv-BGN-transfected BGN-KO osteoblastic cells have a higher binding affinity to BMP-4 than Adv-BGNm-transfected cells. Images of BMP-4-positive cells were captured by a fluorescence microscope. The green fluorescence shown in 1, 2 and 3 represents BMP-4-positive cells. The blue fluorescence shown in 4, 5 and 6 represents nuclear staining with Hoechst corresponding to 1, 2 and 3. (B) Quantitative analysis of the fluorescence intensity of BMP-4-positive cells. "P<0.05, Adv-BGN/Adv-BGNm; ${ }^{\#} \mathrm{P}<0.05$, Adv-BGN/Adv-Emp.

\section{Discussion}

Although the ability of BGN to regulate osteoblast differentiation has been known for many years, the details of the underlying mechanism have not been fully elucidated. In the present report, we studied the effects of GAG chains of BGN on the BMP-4-induced osteoblast differentiation. Our results suggest that the GAG chains of BGN act as a positive modulator of BGN activity and promote BMP-4-induced osteoblast differentiation. In particular, immunofluorescence revealed that the expression of BGN in BGN-KO osteoblasts enhanced the cellular affinity to BMP-4 compared to cells expressing non-glycanated BGN (Adv-BGNm), indicating the importance of GAG chains in promoting binding of osteoblastic cells to BMP-4 and osteoblastic differentiation. In vitro ALP activity and Smad1/5/8 phosphorylation demonstrated that Adv-BGNm could not rescue BGN-assisted BMP-4 function and signaling as efficiently as the glycanated BGN (Adv-BGN). It is conceivable that the 2 long GAG chains accommodate more interaction sites for the basic protein BMP-4, thus enhancing the interaction between BGN and BMP-4. Notably, Adv-BGNm transfection of BGN-KO osteoblastic cells treated with BMP-4 significantly enhanced the ALP activity and binding affinity to BMP-4 compared with Adv-Emp transfection, which suggests that other BGN components, such as protein core, may also function to promote BMP-4-induced osteoblast differentiation.

Previous reports support our findings; Miyazaki et al (23) reported that hypersulfated chondroitin sulfate (CS)-E binds to BMP-4 and enhances osteoblast differentiation by increasing the level of exogenous sulfated GAGs to MC3T3-E1 osteo-
A

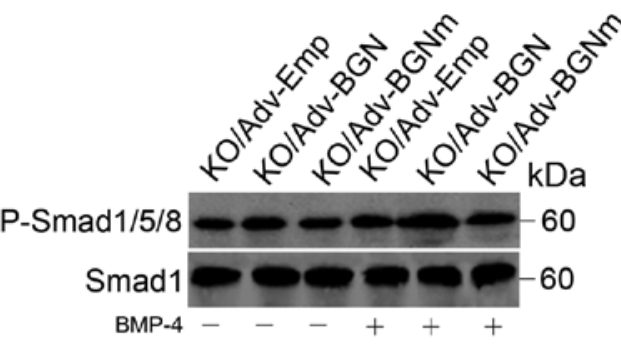

B

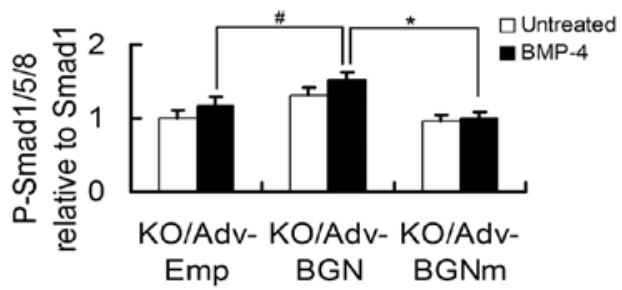

Figure 5. Expression of P-Smad1/5/8 and total Smad-1 in Adv-BGNtransfected BGN-KO osteoblastic cells was significantly higher than that in Adv-Emp-transfected cells and Adv-BGNm-transfected cells. (A) Lanes 1-3 show the level of P-Smad1/5/8 and total Smad-1 in BGN-KO osteoblastic cells transfected with Adv-Emp, Adv-BGN, or Adv-BGNm without BMP-4 treatment; lanes 4-6 show the level of P-Smad1/5/8 and total Smad-1 in BGN-KO osteoblastic cells transfected with Adv-Emp, Adv-BGN and Adv-BGNm and treated with BMP-4. The level of P-Smad1/5/8/Smad-1 in Adv-BGN-transfected cells was significantly higher than that in Adv-Empand Adv-BGNm-transfected cells. ${ }^{~ P}<0.05$, Adv-BGN/Adv-BGNm; ${ }^{*} \mathrm{P}<0.05$, Adv-BGN/Adv-Emp.

blastic cells. It has also been reported that collagen chondroitin sulfate promotes the in vitro mineralization of 3-dimensional collagen matrices seeded with bone-derived cells (24). However, contrary to our present results, a recent report suggested that the GAG component of BGN suppresses the BGN-assisted BMP-2 function in mouse C2C12 myoblastic cells (25). The apparently different functions of GAGs in C2C12 myoblastic cells and BGN-KO osteoblastic cells could be due to the fact that $\mathrm{C} 2 \mathrm{C} 12$ myoblastic cells and BGN-KO osteoblastic cells are at different differentiation stages. Another possible explanation is that GAGs may have different effects on different BMP members. The differences in testing methods and the biological differences between these 2 cell lines should also be considered as explanations for the discrepancies between these results.

It is well known that the Smad pathway is active during osteoblast differentiation. When BGN-KO osteoblastic cells were transfected with Adv-BGN, Smad1/5/8 phosphorylation, ALP activity, and the expression of the transcription factor Cbfa1 and related proteins were all upregulated, indicating that GAGs may facilitate osteoblast differentiation through Smad1/5/8 phosphorylation and Cbfa1 activity. This finding indicates that the GAGs are essential for BGN to regulate Cbfa1 transcriptional activity. A related report showed that glycanated BGN is able to increase the phosphorylation of Smad1/5/8 (26). Markedly, it was reported that the levels of non-glycanated forms of BGN increase with age in human articular cartilage (27). A similar trend was observed in both articular cartilage and intervertebral discs. Therefore, we hypothesize that in early development (i.e., infancy, childhood and adolescence), osteoblast differentiation is quite active 
and that the body needs more glycanated forms of BGN to enable BMP-4 to execute its normal biological functions, which require the GAG chains of BGN. Furthermore, we hypothesize that in adulthood, the body is fully developed and osteoblast differentiation becomes relatively inactive. Therefore, the GAG chains of BGN may be less essential during adulthood than during childhood, and this could be reflected by the increase in the levels of non-glycanated BGN.

In conclusion, our study demonstrated that GAG chains increase $\mathrm{BGN}$-assisted BMP-4 signaling and osteoblast differentiation in BGN-KO osteoblastic cells, demonstrating the positive effect of GAG chains of BGN on BMP-4-induced osteoblast differentiation. This finding represents an important step towards the development of new treatments for bone diseases and BGN-related disorders.

\section{Acknowledgements}

This study was supported by the National Natural Science Foundation of China (nos. 30650006 and 31070831) and the PhD Program Foundation of the Ministry of Education of China (no. 20050487061).

\section{References}

1. Iozzo RV: The biology of the small leucine-rich proteoglycans. Functional network of interactive proteins. J Biol Chem 274: 18843-18846, 1999.

2. Ameye L and Young MF: Mice deficient in small leucine-rich proteoglycans: novel in vivo models for osteoporosis, osteoarthritis, Ehlers-Danlos syndrome, muscular dystrophy, and corneal diseases. Glycobiology 12: 107R-116R, 2002.

3. Roughley PJ and White RJ: Dermatan sulphate proteoglycans of human articular cartilage. The properties of dermatan sulphate proteoglycans I and II. Biochem J 262: 823-827, 1989.

4. Bianco P, Fisher LW, Young MF, Termine JD and Robey PG: Expression and localization of the two small proteoglycans biglycan and decorin in developing human skeletal and nonskeletal tissues. J Histochem Cytochem 38: 1549-1563, 1990.

5. Young MF, Bi Y, Ameye L and Chen XD: Biglycan knockout mice: new models for musculoskeletal diseases. Glycoconj J 19 257-262, 2002

6. $\mathrm{Xu} \mathrm{T}$, Bianco P, Fisher LW, et al: Targeted disruption of the biglycan gene leads to an osteoporosis-like phenotype in mice. Nat Genet 20: 78-82, 1998.

7. Chen XD, Shi S, Xu T, Robey PG and Young MF: Age-related osteoporosis in biglycan-deficient mice is related to defects in bone marrow stromal cells. J Bone Miner Res 17: 331-340, 2002.

8. Chen XD, Fisher LW, Robey PG and Young MF: The small leucine-rich proteoglycan biglycan modulates BMP-4-induced osteoblast differentiation. FASEB J 18: 948-958, 2004.

9. Moreno M, Munoz R, Aroca F, Labarca M, Brandan E and Larrain J: Biglycan is a new extracellular component of the Chordin-BMP4 signaling pathway. EMBO J 24: 1397-1405, 2005 .
10. Bernfield M, Gotte M, Park PW, et al: Functions of cell surface heparan sulfate proteoglycans. Annu Rev Biochem 68: 729-777, 1999.

11. Casu B and Lindahl U: Structure and biological interactions of heparin and heparan sulfate. Adv Carbohydr Chem Biochem 57: 159-206, 2001.

12. Seidler DG and Dreier R: Decorin and its galactosaminoglycan chain: extracellular regulator of cellular function? IUBMB Life 60: 729-733, 2008.

13. Timmer NM, Kuiperij HB, de Waal RM and Verbeek MM: Do amyloid beta-associated factors co-deposit with Abeta in mouse models for Alzheimer's disease? J Alzheimers Dis 22: 345-355, 2010.

14. Scott JE: Supramolecular organization of extracellular matrix glycosaminoglycans, in vitro and in the tissues. FASEB J 6: 2639-2645, 1992.

15. Nikitovic D, Zafiropoulos A, Tzanakakis GN, Karamanos NK and Tsatsakis AM: Effects of glycosaminoglycans on cell proliferation of normal osteoblasts and human osteosarcoma cells depend on their type and fine chemical compositions. Anticancer Res 25: 2851-2856, 2005.

16. Bouvier M, Couble ML, Hartmann DJ, Gauthier JP and Magloire H: Ultrastructural and immunocytochemical study of bone-derived cells cultured in three-dimensional matrices: influence of chondroitin-4 sulfate on mineralization. Differentiation 45: 128-137, 1990.

17. Chen XD, Qian HY, Neff L, Satomura K and Horowitz MC: Thy-1 antigen expression by cells in the osteoblast lineage. J Bone Miner Res 14: 362-375, 1999.

18. McCarthy TL, Centrella M and Canalis E: Further biochemical and molecular characterization of primary rat parietal bone cell cultures. J Bone Miner Res 3: 401-408, 1988.

19. Komori T, Yagi $\mathrm{H}$ and Nomura $\mathrm{S}$, et al: Targeted disruption of Cbfa1 results in a complete lack of bone formation owing to maturational arrest of osteoblasts. Cell 89: 755-764, 1997.

20. Ducy P, Zhang R, Geoffroy V, Ridall AL and Karsenty G: Osf2/ Cbfa1: a transcriptional activator of osteoblast differentiation. Cell 89: 747-754, 1997.

21. Karsenty G: The genetic transformation of bone biology. Genes Dev 13: 3037-3051, 1999.

22. Parisuthiman D, Mochida Y, Duarte WR and Yamauchi M: Biglycan modulates osteoblast differentiation and matrix mineralization. J Bone Miner Res 20: 1878-1886, 2005.

23. Miyazaki T, Miyauchi S, Tawada A, Anada T, Matsuzaka S and Suzuki O: Oversulfated chondroitin sulfate-E binds to BMP-4 and enhances osteoblast differentiation. J Cell Physiol 217: 769-777, 2008.

24. Schonherr E, Witsch-Prehm P, Harrach B, Robenek H Rauterberg $\mathbf{J}$ and Kresse $\mathrm{H}$ : Interaction of biglycan with type I collagen. J Biol Chem 270: 2776-2783, 1995.

25. Miguez PA, Terajima M, Nagaoka H, Mochida Y and Yamauchi M: Role of glycosaminoglycans of biglycan in BMP-2 signaling. Biochem Biophys Res Commun 405: 262-266, 2011.

26. Wang X, Harimoto K, Xie S, Cheng H, Liu J and Wang Z: Matrix protein biglycan induces osteoblast differentiation through extracellular signal-regulated kinase and Smad pathways. Biol Pharm Bull 33: 1891-1897, 2010.

27. Roughley PJ, White RJ, Magny MC, Liu J, Pearce RH and Mort JS: Non-proteoglycan forms of biglycan increase with age in human articular cartilage. Biochem J 295: 421-426, 1993. 